\title{
COARCTATION OF THE AORTA WITH RIGHT AORTIC ARCH AND ISOLATION OF THE LEFT INNOMINATE ARTERY: A SURGICAL CHALLENGE IN A PATIENT WITHOUT COLLATERAL POSTERIOR BRAIN CIRCULATION
}

\author{
C. Delgado, MD, PhD, and F. Barturen, MD, PhD, Palma de Mallorca, Spain
}

D'Cruz, ${ }^{1}$ Levine, ${ }^{2}$ and their associates in 1966 described the first cases of right aortic arch and isolation of the left innominate artery from the aorta. To date, only 11 previously reported cases have been documented. ${ }^{1,3}$ Coarctation of the aorta

From the Department of Internal Medicine, Service of Cardiology and Intensive Care Unit, Policlínica Miramar, Palma de Mallorca, Spain.

Received for publication May 15, 1998; accepted for publication May 20, 1998

Address for reprints: Carlos Delgado, MD, PhD, Servicio de Cardiología, Policlínica Miramar, Camino de la Vileta, 30, Baleares, Palma de Mallorca 07011, Spain.

J Thorac Cardiovasc Surg 1998;116:657-9

Copyright $\odot 1998$ by Mosby, Inc.

0022-5223/98 $\$ 5.00+0 \quad \mathbf{1 2 / 5 4 / 9 1 8 4 5}$ with right-sided aortic arch is rare. A search of the surgical literature revealed only 7 reported cases. ${ }^{4}$ In this report we describe a 5-year-old girl with severe coarctation of the aorta, right aortic arch, and isolation of the left innominate artery. Associated agenesis of the posterior communicant arteries in the circle of Willis was demonstrated by angiography. Because of the absence of collateral protection of the left cerebellum, we elected not to perform an aortic repair.

Clinical summary. A symptom-free 5-year-old girl was referred to our center for evaluation of a heart murmur. The examination included the following blood pressures in millimeters of mercury: right arm 85/40; left arm 90/45; both lower extremities $95 / 50$. A grade $2 / 6$ midsystolic murmur was heard at the left sternal border radiating to the neck, and a grade 3/6 long systolic murmur was heard over the interscapular area. The peripheral and central pulses (graded on a scale of

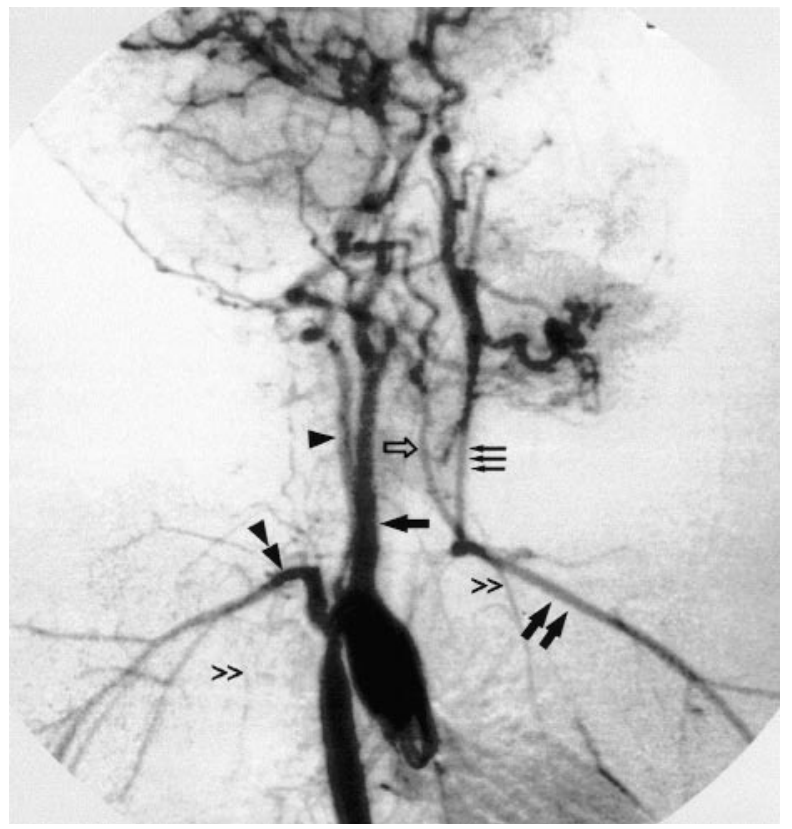

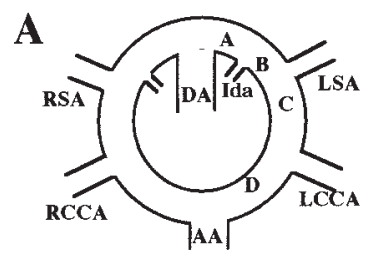

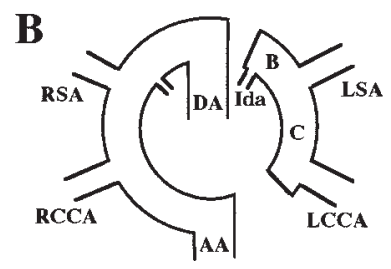

C

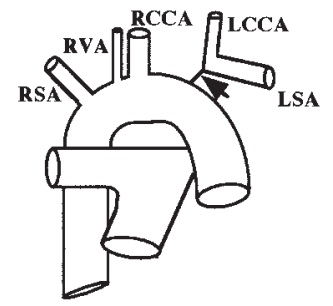

Fig 1. Left, Aortic arch angiogram in right anterior oblique position. The film demonstrates a right-sided aortic arch with 3 vessels arising from the arch in the following order: right common carotid (straight arrow), right vertebral (arrowhead), and right subclavian (doubled arrowhead) arteries. Delayed retrograde opacification of the left vertebral (open arrow), left common carotid (small arrows), and left subclavian (double arrow) isolated arteries were observed on the same film. Underdevelopment of the right and left thoracic arteries can also be seen (〉). Catheter tip is in ascending aorta. Right, Diagram showing the development of type AD right aortic arch: A, The hypothetical double arch of Edwards. ${ }^{5} \mathbf{B}$, Interruption of the left arch in zones A and D. C, Transformation of the left arch into an isolated left innominate artery (arrow). AA, Ascending aorta; DA, descending aorta; $L C C A$, left common carotid artery; RCCA, right common carotid artery; Ida, left ductus arteriosus; LSA, left subclavian artery; $R S A$, right subclavian artery; $R V A$, right vertebral artery. 


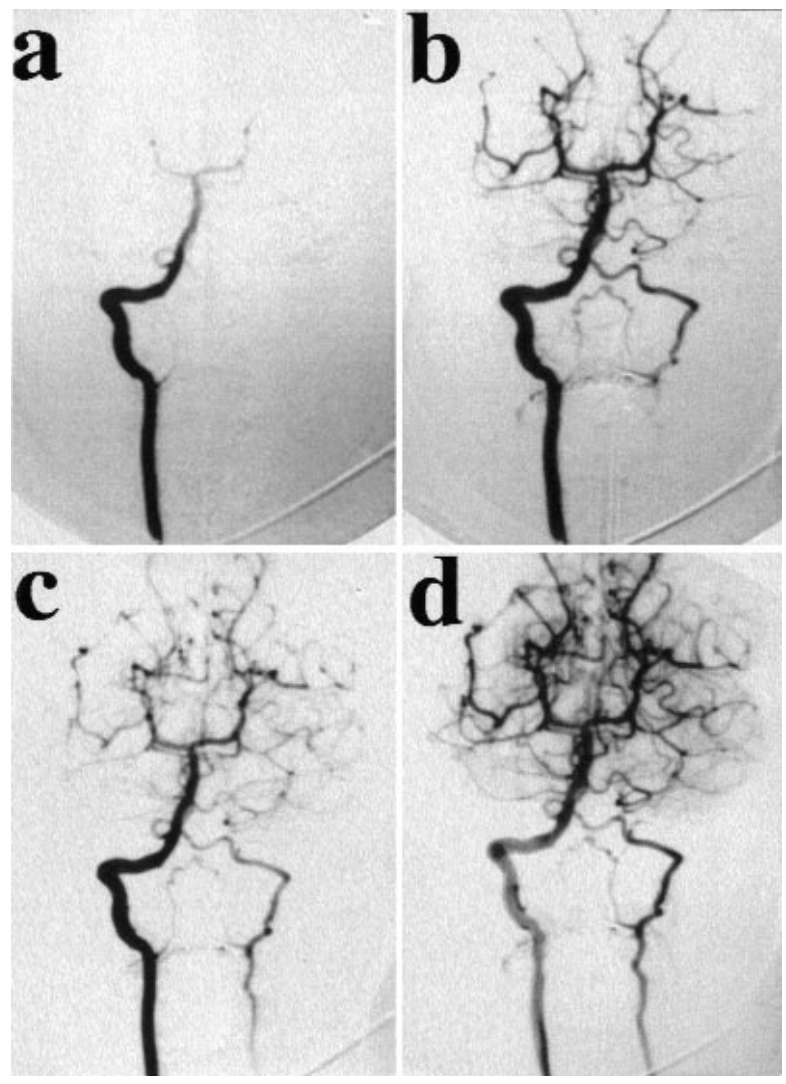

Fig 2. Sequential posteroanterior angiograms with catheter tip in proximal right vertebral artery $(\mathbf{a}, \mathbf{b}, \mathbf{c}$, and $\mathbf{d})$. The films demonstrate retrograde opacification of left vertebral artery across the vertebrobasilar junction and by muscular collateral vessels in the neck. The absence of a posterior communicating artery can also be suspected.

0 -4) were as follows: right carotid +4 ; left carotid +1 ; right brachial +1 ; left brachial +1 ; both femoral and distal lower extremity pulses +1 . A frontal chest $x$-ray film demonstrated a right aortic arch. There was no rib notching on either side. The barium-filled esophagus demonstrated a right-sided impression at the level of the aortic arch without a posterior impression.

A suprasternal transthoracic echocardiographic approach showed an enlarged right-sided aortic arch. No vessels were found arising from the proximal portion of the arch; from the distal arch 3 vessels arose; the first and third trunks, in order of appearance, corresponded to the right common carotid and right subclavian arteries, respectively. The second trunk, ascending in parallel to the first one, was later identified, after angiography, as the right vertebral artery. In addition, a 12mm-long, 4-mm-diameter segment of aortic coarctation was present. The origin of the right subclavian artery was included at the site of the coarctation. Doppler interrogation of the coarctation revealed a peak flow and mean flow systolic gradient of $91 \mathrm{~mm} \mathrm{Hg}$ and $42 \mathrm{~mm} \mathrm{Hg}$, respectively.

Angiography of the ascending aorta showed a right aortic arch and right-sided descending aorta (Fig 1, left). An aortic diverticulum was not present. The right common carotid, right vertebral, and right subclavian arteries arose in turn from the arch. Delayed opacification of the left vertebral, left common carotid, and left subclavian arteries was observed on the same film. These arteries did not fill from the arch. After a delay of several seconds, the left vertebral artery filled in a retrograde fashion via the circle of Willis and thyrocervicalis collaterals. The left common carotid artery filled retrogradely via maxillary collateral vessels from the right external carotid artery and via an anterior communicating artery from the right internal carotid artery. The left subclavian artery filled from the left vertebral artery. It was apparent that these vessels extended from a common trunk (the left innominate artery) without connection to the aorta. Selective injection of the right vertebral artery (Fig 2) confirmed a subclavian steal from an isolated left subclavian artery. In addition to the congenital isolation of a left innominate artery, no posterior communicating arteries in the circle of Willis were found. Thus the brain stem, cerebellum, and occipital lobes were supplied only by the antegrade flow from the right vertebral artery, whereas the rest of the brain of both cerebral hemispheres depended on the flow from the right internal carotid artery.

Invasive and simultaneous blood pressure recordings obtained by femoral and left radial catheters showed the following blood pressures in millimeters of mercury: ascending aorta 211/137; left radial artery 141/109; right femoral artery 138/110. Right brachial blood pressure obtained by noninvasive monitoring was $135 / 94 \mathrm{~mm} \mathrm{Hg}$. No significant difference between blood pressure recorded in the arms and in the legs was found. A gradient of $73 \mathrm{~mm} \mathrm{Hg}$ measured by pullback was obtained in the coarctated area.

Surgical correction of the coarctation was not attempted for fear of compromising the posterior circulation to the brain. Furthermore, as a result of the origin of both subclavian arteries, the right one from a coarctated area and the left one from the vertebrobasilar junction, the normal collateral circulation usually seen in the typical aortic coarctation was underdeveloped. Therefore, in this case, the risk of postoperative paraplegia was also increased.

Edwards, ${ }^{5}$ using a hypothetical model of double aortic arch, was able to relate the various forms of aortic arch systems to either atresia or disappearance of certain parts of the embryonic pattern. By means of the same principle, it is possible to classify the various forms of right aortic arch in a logical order, depending on the site of interruption of the left arch. The zones of the left aortic arch are labeled $A, B, C$, and $D$ (Fig 1, right). Interruption of the left aortic arch in one or two of these zones may result in various anatomic types of right aortic arch. Accordingly, they may be named type A, B, $\mathrm{C}$, and $\mathrm{D}$, when the interruption is in 1 zone, and type $\mathrm{AB}$, $\mathrm{AC}, \mathrm{AD}, \mathrm{BD}$, and $\mathrm{CD}$, when the interruption is in 2 zones. In our case the interruption of the left arch at the region proximal to the carotid artery (zone D) and also distal to the subclavian artery (zone A) results in complete isolation of these 2 vessels of the aorta. The absence of a posterior indentation in the barium-filled esophagus and the lack of filling of an aortic diverticulum in the aortogram was indicative of type $\mathrm{AD}$ right aortic arch. In this case the left subclavian artery 
steals the blood flow from the right side through the cervical and cerebral anastomotic channels and may cause symptoms of vertebrobasilar insufficiency.

The patient did not have symptoms of cerebral ischemia or significant differences in blood pressure readings between arms. The origin of the right subclavian artery from the aorta in the coarctated region, and the pulse wave attenuation in the circle of Willis of the blood flowing from the right vertebral and right carotid arteries, were the causes of the unexpected equalization of the blood pressure in the 2 arms. In addition, no significant difference between blood pressure recorded in the arms and legs was found in the clinical examination. Only the presence of a weak pulse of the left common carotid artery could have been partially useful in the clinical diagnosis of this anomaly.

In summary, we describe an Edwards's type $\mathrm{AD}^{5}$ right aortic arch in a patient with a severe aortic coarctation. So far as we know, this association has not previously been documented. Because of the absence of the posterior communicating arteries in the circle of Willis, the circulation of the posterior part of the brain depended on the antegrade flow from the right vertebral artery. The anterior part of the brain was supplied only by the flow from the right internal carotid artery. Furthermore, the underdevelopment of the thoracic collateral circulation found in this patient increased the risk of postoperative paraplegia. For these reasons this patient has not had a surgical repair.

We are grateful to Drs Juan Mestre and Javier Pueyo for their valuable contribution.

\section{REFERENCES}

1. D'Cruz JA, Cantez T, Namin EP, et al. Right aortic arch, right descending aorta, and associated anomalies. Br Heart J 1966; 28:725-39.

2. Levine S, Serfas LS, Rusinko A. Right aortic arch with subclavian steal syndrome (atresia of left common carotid and left subclavian arteries). Am J Surg 1966;111:632-7.

3. Moes CA, Freedom RM. Rare types of aortic arch anomalies. Pediatr Cardiol 1993;14:93-101.

4. Moreno-Cabral RJ, Maky HS. Coarctation of aorta with rightsided aortic arch: surgical correction through right thoracotomy [letter]. J Thorac Cardiovasc Surg 1994;108:587-96.

5. Edwards JE. Anomalies of derivatives of aortic arch system. Med Clin North Am 1948;32:925-49.

\section{Commentary}

In this case report, Delgado and Barturen have described a 5-year-old patient with an extremely unusual combination of aortic arch anomalies and anomalies of the circle of Willis. There is no connection from the aortic arch to the left common carotid and left subclavian arteries, which represent an isolated segment of a left aortic arch. In addition, there is a severe coarctation that incorporates the origin of the right subclavian artery from the right-sided aortic arch. There is absence of a complete circle of Willis, so that the posterior cerebral circulation is separate from the anterior cerebral circulation. These findings illustrate nicely the embryology of aortic arch development, as well as the potential for arch stenosis (coarctation) or interruption to occur at points of junction of the various segments of the developing aortic arches.

Delgado and Barturen felt uncomfortable in offering surgical repair to this girl because of their concern regarding a high risk of either cerebral or spinal cord ischemic injury. However, the patient has a left ventricular pressure of greater than $200 \mathrm{~mm}$. The coronary arteries are exposed to a pressure of 211/137 mm Hg. Furthermore, the anterior cerebral circulation is entirely dependent on the right common carotid artery, and there is a steal into the left common carotid system through the circle of Willis. The posterior cerebral circulation is entirely dependent on the right vertebral artery with a steal into the left subclavian system. Although the risks of surgery are undoubtedly greater than the usual risks for coarctation repair, the various problems described above in my opinion justify the risks of surgical repair. I recommend to the authors that they consider the use of deep hypothermic circulatory arrest with a combination of both surface and core cooling. A connection should be established between the proximal aortic arch and the left subclavian/carotid system. Perhaps this could be achieved by turning down the left subclavian artery, although this might risk the viability of the left arm. An alternative would be to insert a polytetrafluoroethylene interposition tube graft. The coarctation should be dealt with by resection and end-to-end anastomosis, if possible, including reimplantation of the right subclavian artery. This procedure should reduce left ventricular pressure and ascending aortic pressure to a normal level and should provide bilateral supply to the anterior and posterior cerebral circulation.

Richard A. Jonas, MD Boston, Mass 12/54/92471 